\title{
Combined Orthodontic and Surgical Treatment of Impacted Maxillary Canine in Young Patient with Class II Malocclusion: a Case Report
}

\author{
Ljubica Pašagić,, Ivana llić,' Vesna Kecman,, ${ }^{2}$ Marko Bulajić, ${ }^{2}$ Nina Zubović,, \\ Branislav Glišić ${ }^{4}$
}

\begin{abstract}
The impaction of maxillary canines is one of the biggest challenges in orthodontics practice. This case report describes successful surgical and orthodontic approach to the treatment of palatally impacted maxillary canine in a 14-year-old boy. Intraoral clinical examination revealed an absence of the upper right canine, an ectopic position of the upper left canine and crowding in the maxillary arch. The impaction of right maxillary canine and class II malocclusion were confirmed by lateral cephalogram, orthopantomogram and cone beam computed tomography. In the first phase, a transpalatal arch to the upper first molar teeth was applied, first premolars were extracted, and brackets were placed on all teeth and nickel-titanium arch wire was applied. The initial orthodontic phase was soon thereafter followed by the surgical exposure and orthodontic traction of the impacted canine using ligature wire attached from the button with chain to the open coil on the arch wire. The orthodontic treatment took two years with satisfactory aesthetic and functional results at the end. This clinical case has shown that adequate treatment of impacted maxillary canine can be achieved by using combined surgical technique and appropriate orthodontic approach.
\end{abstract}

Key words: Impacted canine; Malocclusion, Class II; Orthodontic-surgical approach; Canine traction.
(1) Department of Orthodontics, Dental Clinic, Faculty of Medicine, University of Banja Luka, Banja Luka, the Republic of Srpska, Bosnia and Herzegovina.

(2) Department of Oral Surgery, Dental Clinic, Faculty of Medicine, University of Banja Luka, Banja Luka the Republic of Srpska, Bosnia and Herzegovina

(3) Department of Periodontal Diseases, Dental Clinic, Faculty of Medicine, University of Banja Luka, Banja Luka, the Republic of Srpska, Bosnia and Herzegovina.

(4) Department of Orthodontics, School of Dental Medicine, University of Belgrade, Belgrade, Serbia.

Correspondence: LJUBICA PAŠAGIĆ

E: ljubica.pasagic@med.unibl.org M: +387 65950044

\section{ARTICLE INFO}

Received: 14 September 2020 Revision received: 20 September 2020 Accepted: 20 September 2020

\section{Introduction}

The presence of canines in dental arch are extremely important for facial harmony, smile aesthetic and stabile occlusion. Many factors can influence the functional and aesthetic balance, including the canine impaction. The impaction of the maxillary permanent canines is the second most common form of tooth impaction after the third molars with the rate that varies from 0.2 to $2.8 \%{ }^{1,2}$ Girls are much more frequently affected than boys, with the prevalence ratio from $1.3: 1$ to $3.2: 1$, and with bilateral occlusion occurring in $8 \%$ of cases. ${ }^{3}$ The impacted canines occur 10 -
20 times more frequently in the upper jaw than in the lower jaw 4 and the impaction occurs more frequently in the palatine region than in the buccal one (85\% vs $15 \%){ }^{5}$

The causes of the maxillary canine impaction can be classified into several distinct groups: local tissue obstruction, local pathology, disturbance of normal development and hereditary or genetic factors. ${ }^{4}, 6$ Canine impaction is very often accompanied by the persistence of deciduous teeth, cysts or dental ankyloses. ${ }^{7}$ 
There are several options for treatment of maxillary canine impaction. Some of them include extraction followed by an implant support or a prosthetic replacement procedure. However, if the position of impacted canine in the bone allows an orthodontic-surgical method, the orthodontic treatment should be started as soon as possible to avoid secondary problems. ${ }^{8}$ The treatment proce- dure is based on surgical release and orthodontic traction of an impacted canine to the dental arch.

The aim of this report is to describe the orthodontic treatment in patient with impacted maxillary permanent canine using a combined surgical-orthodontic approach, and to validate the functional and aesthetic results.

\section{Case history}

A 14-year-old male patient was referred to the Department of Orthodontics, Dental Clinic of the Faculty of Medicine, University of Banja Luka, the Republic of Srpska, Bosnia \& Herzegovina, for orthodontic consultation related to aesthetic reasons. The extraoral clinical examination revealed that the patient's face was symmetric with convex profile and unaesthetic smile. During intraoral clinical examination the absence of upper right canine, ectopic position of upper left canine and crowding in the maxillary arch were diagnosed. In the following steps the orthodontic records were taken including maxillary and mandibular impressions, extraoral and intraoral photographs, lateral cephalogram, panoramic radiograph and cone beam computed tomography (CBCT) of the maxilla (Figures 1 and 2). Gnatometric analyses of the study models revealed the Class II malocclusion. The panoramic radiograph showed the presence of all permanent teeth in-

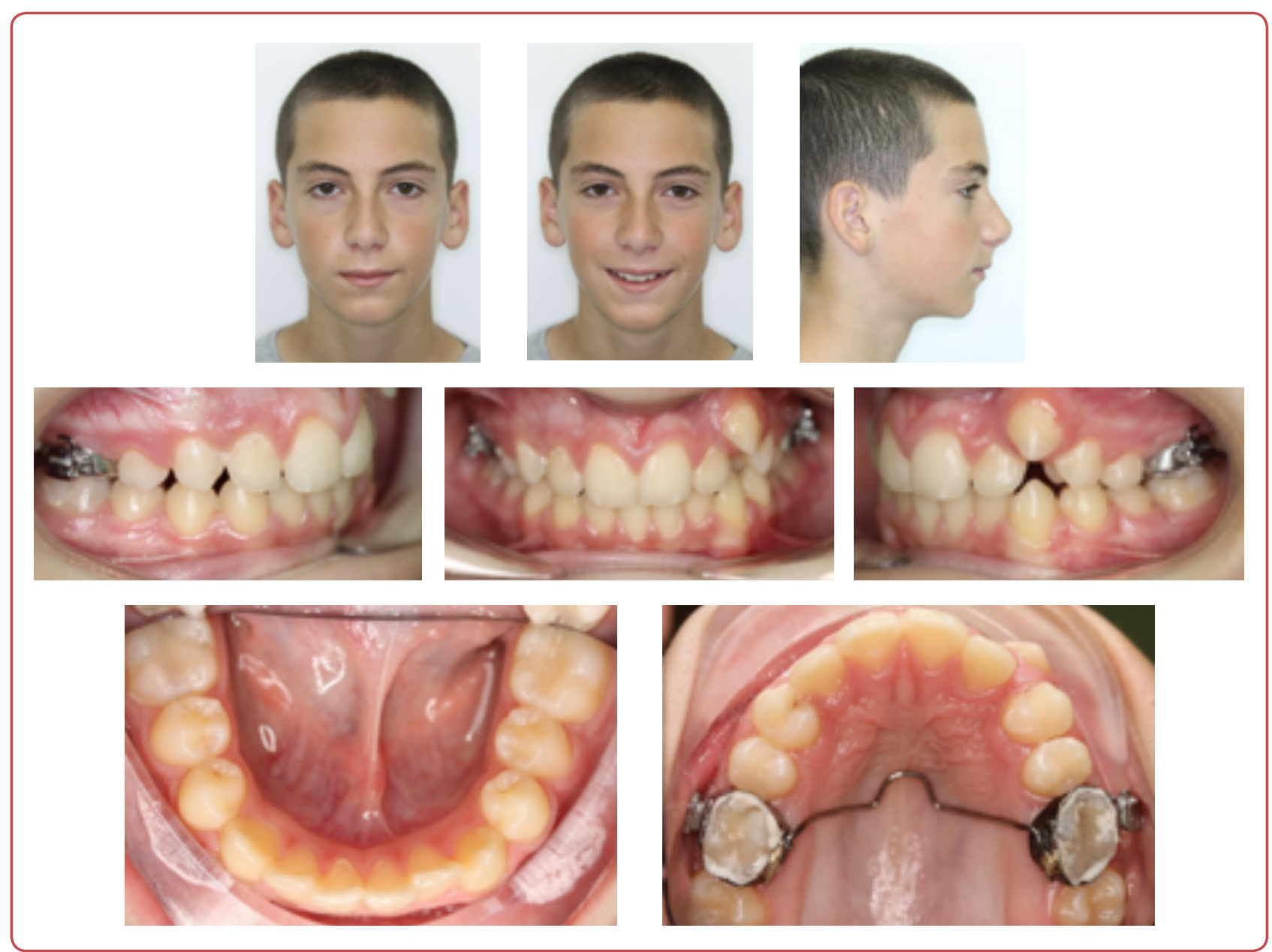

Figure 1: Extraoral and intraoral pretreatment photographs. 


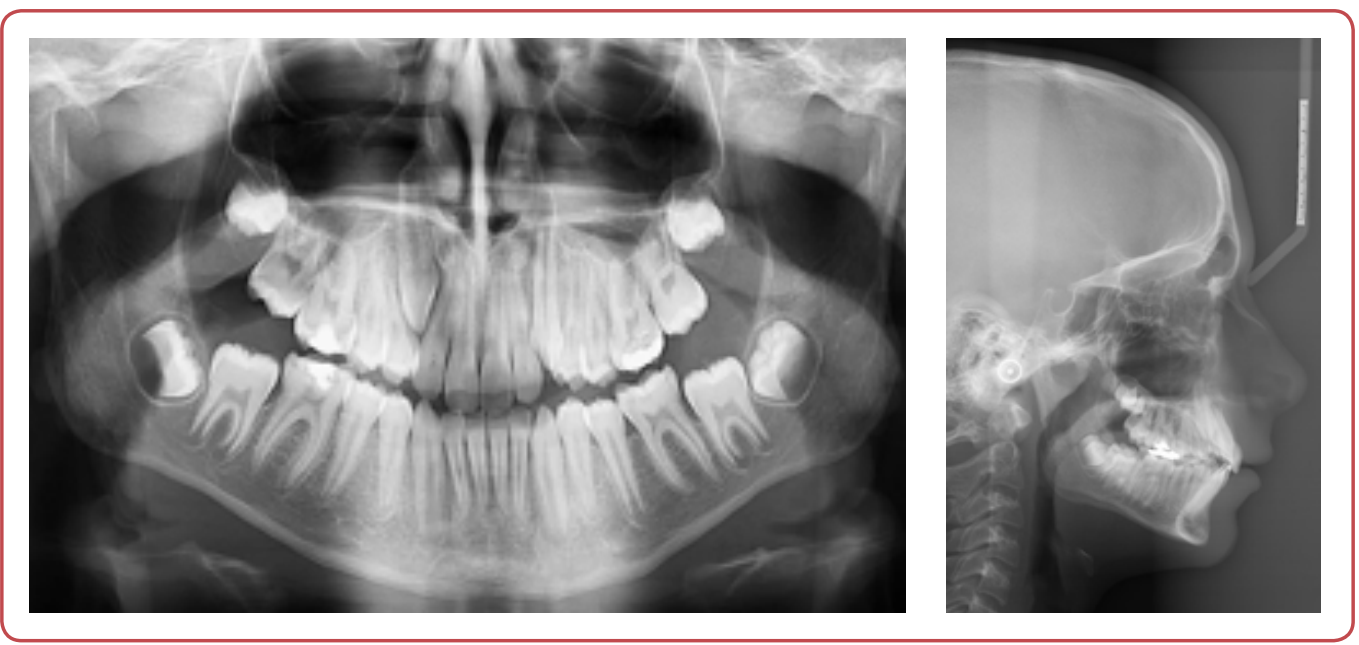

Figure 2: Initial radiographs; panoramic radiography (left) and lateral teleradiography (right).

cluding the developing third molar buds and intraosseal impaction of right permanent canine in the maxilla (Figure 2). The orthopantomogram analysis revealed an increased angle between the maxillary canine and lateral incisors indicating mesial inclination (Figure 2). The CBCT of maxilla demonstrated that the canine was impacted on palatal site. The cephalometric analysis on the lateral cephalogram confirmed a skeletal Class II malocclusion. After the diagnostic analyses was completed, the patient was scheduled for the combined surgical-orthodontic treatment.

\section{Course of treatment}

After a detailed analysis of the study model was done, the surgical and orthodontic treatment was applied. In the initial orthodontic phase the transpalatal arch to the upper first molar teeth was applied to maintain an anchorage (Figure 1). After that, the first premolars were extracted, brackets were placed on all teeth and a 0.012 nickel-titanium (NiTi) arch wire was applied. In this case the metal brackets of 0.018 slot were used. Open coil spring was positioned between 1.2 and 1.4 to increase the space for the permanent canine. Levelling and alignment phase was done by wire sequences of $0.012 ", 0.014 ", 0.016 ", 0.018 ", 0.016 " \mathrm{x}$ 0.022 " NiTi, followed by $0.016 " x$ 0.022 " stainless steel arch wire. After orthodontic initial phase was completed the surgical exposure of 1.3 was performed using an open eruption technique. Surgical circular excision of the overlying palatal mucosa was performed and a small amount of bone covering the impacted canine was removed. The enamel surface of the impacted canine was washed and dried and then an orthodontic button with a chain for the guided traction was bonded at the crown level. Three weeks later the patient

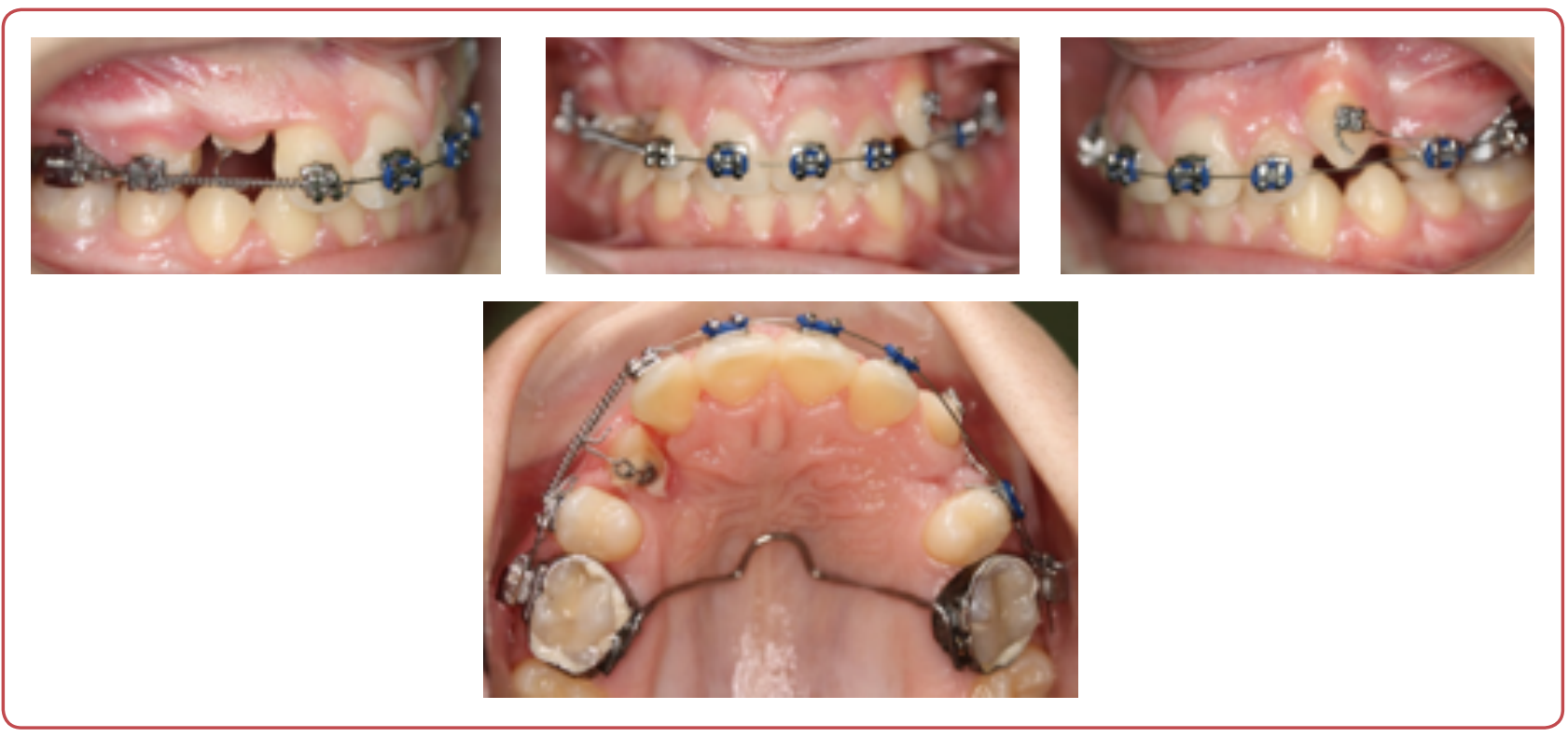

Figure 3: Orthodontic traction of the canine into the dental arch. 


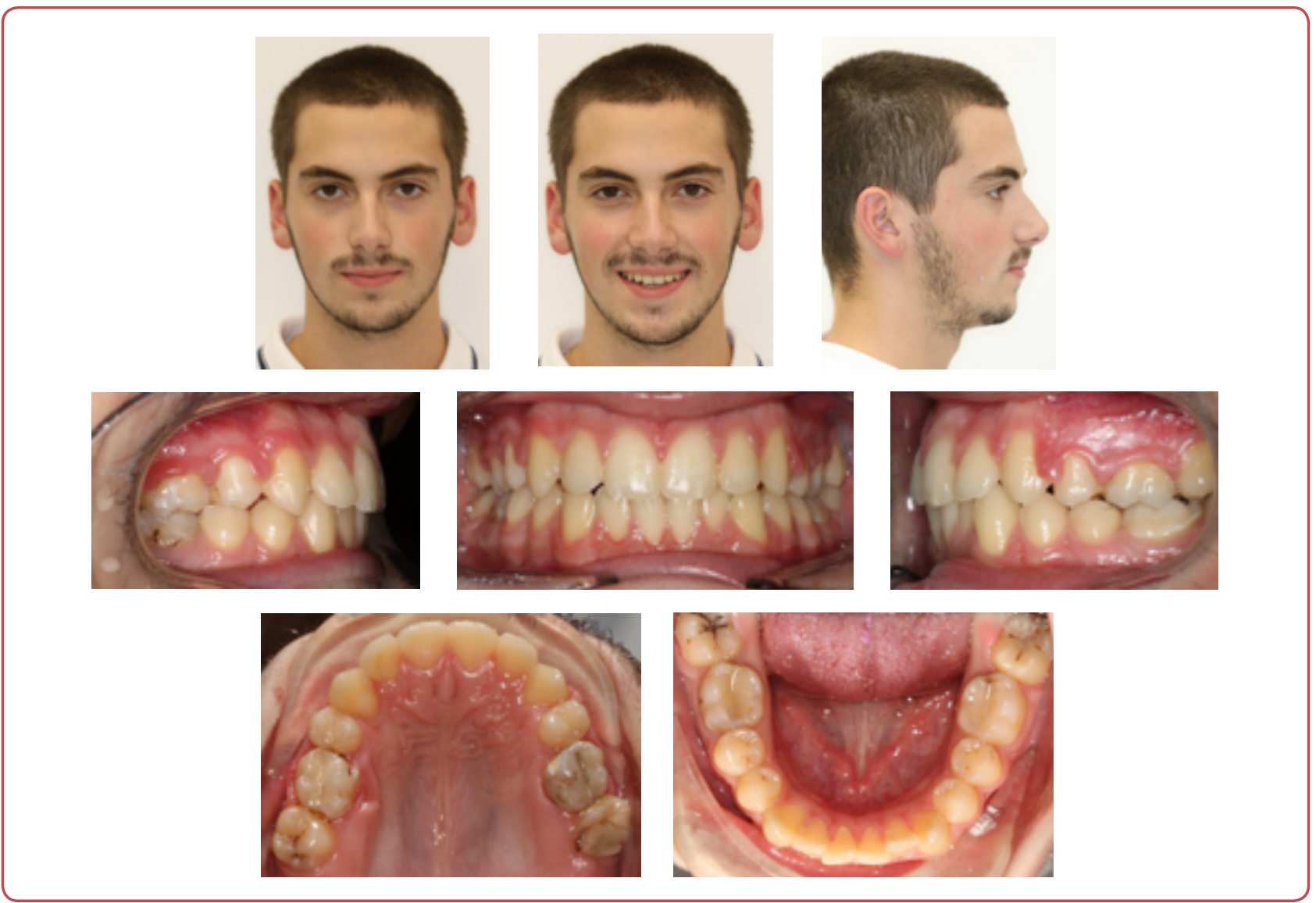

Figure 4: Post-treatment extraoral and intraoral photographs.

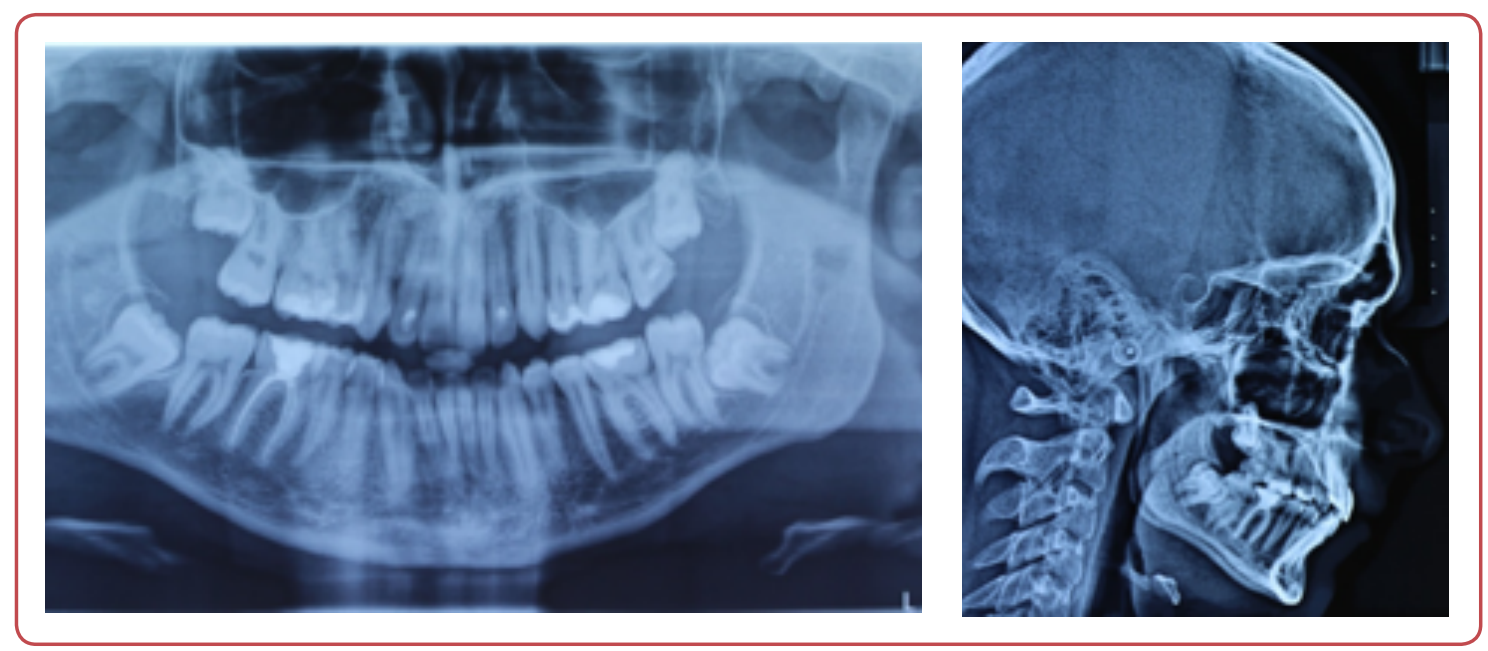

Figure 5: Post-treatment radiographs; panoramic radiography (upper) and lateral teleradiography (right).

returned to the orthodontic department after the soft tissues had healed. Traction of the impacted maxillary canine was made with ligature wire attached from the button with chain to the open coil on the arch wire. The patient came for checkups every three weeks (Figure 3). Eight months later the left maxillary canine was near the arch and the bracket was bonded to the labial surface of its crown and tied to arch wire of 0.016 " NiTi to begin the final orthodontic phase. Maxillary left canine alignment and levelling was done by following wire sequence of $0.018 "$ and $0.016 " \mathrm{x}$ $0.022 "$ NiTi. Finalising was achieved with stainless steel arch wire of $0.016 "$ x $0.022 "$ " The active treatment took 24 months and retention was accomplished with a removable acrylic retainer. The final aesthetic result was satisfactory and the final radiographs indicated intact roots and proper root alignment (Figures 4 and 5). 


\section{Discussion}

The treatment of impacted teeth is among the most complex procedures in orthodontics that requires multidisciplinary approach. In order to identify and locate the position of impacted teeth adequate tomographic images are required. It also helps to evaluate possible injuries to adjacent roots, to estimate the quality and quantity of bone around the teeth, to detect the existence of possible tooth ankylosis and occurrence of follicular cysts or infections. ${ }^{7,9}$ In this clinical case the post-treatment tomographic image evaluation did not show any signs of external root resorption.

Besides the position of the teeth, the patient's age can also determine the type of treatment. ${ }^{10}$ For adult patients the tooth extraction is recommended method of treatment. ${ }^{3}$ However, for younger patients with favourable position of impacted canine, combined surgical and orthodontic treatment is suggested. The best treatment for impacted canine is surgical exposure and orthodontic traction in order to bring the impacted tooth to the line of occlusion using light and slow orthodontic forces. ${ }^{11}$ The movement of $1 \mathrm{~mm}$ per month has been considered as an optimum traction. ${ }^{12}$ However, concerning the surgical exposure it is important to emphasise that good collaboration between the orthodontist and the surgeon is of crucial importance to define and select the most appropriate technique. ${ }^{13}$ Similarly to the spontaneous tooth eruption, the combined surgical and orthodontic approach to the treatment of an impacted canine has to provide conditions to facilitate the physiological eruption. ${ }^{14}$ It has been shown that combined orthodontic-surgical traction of impacted canines provides near- natural conditions for tooth eruption and reduce the need for prosthetic therapy. ${ }^{15}$ This report has described a case of unilateral impaction of permanent maxillary canine in a young male patient. In this case the open eruption method was used in order to reduce the risk of the second surgical exposure. ${ }^{11,16}$

\section{Conclusion}

This clinical case has shown that treatment of impacted maxillary canine can be achieved by using combined surgical technique and appropriate orthodontic approach in order to obtain good aesthetic and functional results. An adequate orthodontic force with optimum traction is a key component in orthodontic treatment needed for satisfactory occlusion and good aesthetic results.

\section{Acknowledgements}

None.

\section{Conflict of interest}

None.

\section{References}

1. Peck S, Peck L, Kataja M. The palatally displaced canine as a dental anomaly of genetic origin. Angle Orthod 1994;64:249-56.

2. Becker A and Chaushu S. Etiology of maxillary canine impaction: a review. Am J Orthod Dentofacial Orthop 2015;148:557-67.

3. Bishara SE. Impacted maxillary canines: a review. Am J Orthod Dentofacial Orthop 1992 Feb;101(2):159-71.

4. Sharma G, Nagpal A. Transmigration of mandibular canine: report of four cases and review of literature. Case Rep Dent 2011; 2011:381382. doi: $10.1155 / 2011 / 381382$.

5. Rutledge MS and Hartsfield JK. Genetic factors in the etiology of palatally displaced canines. Sem Orthodont 2010;16:165-71.

6. Raghav P, Singh K, Reddy CM, Joshi D, Jain S. Treatment of maxillary impacted canine using ballista spring and orthodontic wire traction. Int J Clin Pediatr Dent 2017;10(3):313-7.

7. Araújo EA, Araújo CV, Tanaka OM. Apicotomy: surgical management of maxillary dilacerated or ankylosed canines. Am J Orthod Dentofacial Orthop 2013 Dec;144(6):909-15.

8. Elefteriadis JN, Athanasiou AE. Evaluation of impacted canines by means of computerized tomography. Int J Adult Orthodon Orthognath Surg 1996;11(3):257-64.

9. Pignoly M, Monnet-Corti V, Le Gall M. Reason for failure in the treatment of impacted and retained teeth. Orthod Fr 2016 Mar;87(1):23-38.

10. Kokich VG. Surgical and orthodontic management of impacted maxillary canines. Am J Orthod Dentofacial Orthop 2004;126(3):278-83.

11. Celli D, Catalfamo L, Deli R. Palatally impacted canies: the double traction technique. Prog Orthod 2007;8:1626. 
12. Ferreira JTL, Romano FL, Sasso Stuani MB, Assed Carneiro FC, Nakane Matsumoto MA. Traction of impacted canines in a skeletal Class III malocclusion: A challenging orthodontic treatment. Am J Orthod Dentofacial Orthop 2017 Jun;151(6):1159-68.

13. Cruz RM. Orthodontic traction of impacted canines: concepts and clinical application. Dental Press J Orthod 2019 Jan-Feb;24(1):74-87.

14. Figliuzzi MM, Altilia M, Mannarino L, Giudice A, Fortuna- to L. Minimally invasive surgical management of impacted maxillary canines. Ann Ital Chir 2018;89(5):443-7.

15. Pavlović J, Tabaković SZ, Simić S, Vujačić A, VukićevićV. Orthodontic-surgical treatment of four impacted canines in an adult patient: a case report. Vojnosanit Pregl 2016;73(7):682-5

16. Ferguson J, Parvizi F. Eruption of palatal canines following surgical exposure: a review of outcomes in a series of consecutively treated cases. Br Dent J 1997;24:203-7. 